\title{
HEARING AND HUNTING IN RED BATS (LASIURUS BOREALIS, VESPERTILIONIDAE): AUDIOGRAM AND EAR PROPERTIES
}

\author{
MARTIN K. OBRIST ${ }^{1, *}$ AND JEFFREY J. WENSTRUP ${ }^{2}$ \\ ${ }^{1}$ Swiss Federal Institute for Forest, Snow and Landscape Research, CH-8903 Birmensdorf, Switzerland and \\ ${ }^{2}$ Department of Neurobiology, Northeastern Ohio Universities College of Medicine, Rootstown, OH 44272, USA \\ *e-mail: martin.obrist@wsl.ch
}

Accepted 6 October 1997; published on WWW 9 December 1997

\begin{abstract}
Summary
We examined aspects of hearing in the red bat (Lasiurus borealis) related to its use of biosonar. Evoked potential audiograms, obtained from volume-conducted auditory brainstem responses, were obtained in two bats, and the sound pressure transformation of the pinna was measured in three specimens. Field-recorded echolocation signals were analysed for comparison. The fundamental sonar search calls sweep from 45 to $30 \mathrm{kHz}$ (peak energy at $35 \mathrm{kHz}$ ), approach-phase calls sweep from 65 to $35 \mathrm{kHz}$ (peak $40 \mathrm{kHz}$ ) and terminal calls sweep from 70 to $30 \mathrm{kHz}$ (peak $45 \mathrm{kHz}$ ). The most sensitive region of the audiogram

of the pinna moves upwards and medially. The sound pressure transformation was noteworthy near $40-45 \mathrm{kHz}$; the acoustic axis was closest to the midline, the $-3 \mathrm{~dB}$ acceptance angles showed local minima, and the pinna gain and interaural intensity difference were maximal. These results are related to the known echolocation and foraging behavior of this species and match the spectral components of approach- and final-phase calls. We conclude that coevolution with hearing prey has put a higher selective pressure on optimizing localization and tracking of prey than on improving detection performance.
\end{abstract} extended from $10 \mathrm{kHz}$ to $45-55 \mathrm{kHz}$, with maximum sensitivity as low as $20 \mathrm{~dB}$ SPL occurring between 25 and $30 \mathrm{kHz}$. A relative threshold minimum occurred between 40 and $50 \mathrm{kHz}$. With increasing frequency, the acoustic axis

Key words: Lasiurus borealis, red bat, echolocation, audiogram, hearing, hunting, pinna.

\section{Introduction}

Many foraging vespertilionids use echolocation to detect and track targets (Griffin, 1958), and their vocalization and hearing systems are remarkably well-adapted to the needs of active orientation. Echolocating bats have driven to extremes some or all of the auditory anatomy, physiology and behavior of generalized mammals. Some species are experts in passive listening owing to their large pinnae (e.g. Megadermatidae or Nycteridae; Fenton et al. 1990; Guppy and Coles, 1988), others have evolved cochlear and neuronal adaptations that allow them to detect fluttering targets (Mormoopidae, Rhinolophidae and Hipposideridae; Bell and Fenton, 1984; Henson et al. 1987; Schnitzler, 1987; Schnitzler and Henson, 1980; Schnitzler et al. 1983; Schuller et al. 1974; Von der Emde and Menne, 1989). Most vespertilionid bats appear to have a rather generalized mammalian hearing system, using behavioral flexibility to increase their foraging effectiveness (Kalko and Schnitzler, 1993; Obrist, 1995; Rydell, 1993; Schumm et al. 1991). The use of frequency-modulated (FM) signals of differing degrees of modulation seems to be one key to their evolutionary success. Recent studies of the auditory system have revealed neural specializations related to this behavioral feature in Eptesicus fuscus (Vespertilionidae; Casseday and Covey, 1992) and Tadarida brasiliensis mexicana

(Molossidae; Vater and Siefer, 1995), species that both use FM signals with varying degrees of modulation and very narrowband echolocation calls (Obrist, 1995; Simmons et al. 1978) when searching for insects (Griffin et al. 1960). Both species also exhibit pinna properties relatable to the dominant frequencies of their search-phase echolocation calls (Obrist et al. 1993).

Lasiurus borealis is an insectivorous bat distributed throughout most of the forested part of North America. Individuals roost alone and hunt above or close to trees but rarely within the canopy (Shump and Shump, 1982). Groups of several bats forage and interact near street lights (Hickey and Fenton, 1990), where they feed on an abundance of insects, especially Lepidoptera (Acharya and Fenton, 1992). L. borealis are remarkably flexible in their echolocation behavior, adapting their echolocation calls in temporal and spectral range to their acoustic environment as well as to the presence of conspecifics (Obrist, 1995). Red bats eavesdrop on conspecifics (Balcombe and Fenton, 1988), attracted by their final buzzes emitted with dominant energy at $45 \mathrm{kHz}$. The eavesdropping often leads to chases between bats (Hickey and Fenton, 1990), in which low-frequency vocal interactions are common (M. K. Obrist, personal observation), suggesting that 
lower-frequency bands are used in communication. L. borealis uses a long, final, narrow-band component in its sonar signals when foraging over long distances (Obrist, 1995), but its outer ears do not seem to be tuned to the dominant search-call frequency (Obrist et al. 1993). Here, we test the hypothesis that other characteristics of higher-frequency content of the sonar emissions of this species are reflected in either the auditory sensitivity or the passive acoustic properties of the pinna, or both.

\section{Materials and methods}

Auditory brainstem response (ABR)

Evoked potential audiograms, obtained from volumeconducted auditory brainstem responses (ABRs), were obtained in two red bats Lasiurus borealis (Müller, 1776) caught in Pinery Provincial Park, Ontario, Canada. In one bat, thresholds were obtained on two different days to assess their stability. Recordings of ABR closely followed the method employed by Wenstrup (1984).

\section{Surgical preparation}

To implant the recording electrode, the animal was first anesthetized with Metofane (Pitman-Moore, Inc.). A small incision was made in the skin overlying the dorsal aspect of the cranium. The electrode, a $0.25 \mathrm{~mm}$ diameter silver wire wrapped into a flat spiral, was inserted between the skin and the underlying musculature, centered over the central sagittal plane. The reference electrode, a silver wire wrapped around saline-soaked cotton, was placed in the animal's mouth. During recording, the bat remained lightly anesthetized with Metofane and a cocktail of Nembutal $\left(5 \mathrm{mg} \mathrm{kg}^{-1}\right)$ and acepromazine $\left(5 \mathrm{mg} \mathrm{kg}^{-1}\right)$. The animal's small surgical wound was treated with lidocaine. Both lidocaine and saline were renewed as necessary. The unrestrained, anesthetized bat was placed on a heating pad. Although rectal temperature was not monitored, a thermometer placed between the heating pad and the animal's venter was maintained at $31-33^{\circ} \mathrm{C}$.

\section{Acoustic apparatus}

Recordings were obtained in an acoustic chamber lined with sound-attenuating foam. Surfaces near the bat were also covered with cotton to reduce echoes. An Apple Macintosh IIx computer, running applications developed within National Instruments LabVIEW software, controlled stimulus generation and data acquisition. To generate sounds, the computer controlled a Hewlett-Packard waveform generator (model 8904), whose output was shaped into pulses and attenuated by a Tucker-Davis Technology SW1 switch and PA3 programmable attenuator, respectively. The attenuator output was amplified (Coulbourn model S82-24) and sent to a Panasonic leaf tweeter placed $34 \mathrm{~mm}$ directly in front of the bat. Sound stimuli consisted of $10 \mathrm{~ms}$ tone bursts with $1 \mathrm{~ms}$ rise/fall times, presented a maximum of seven times per second. At frequencies below $5 \mathrm{kHz}, 2 \mathrm{~ms}$ rise/fall times were used to avoid the distorting effects of short rise times. At
$2500 \mathrm{~Hz}, 1 \mathrm{~ms}$ and $2 \mathrm{~ms}$ rise/fall times yielded identical threshold values.

The speaker output was calibrated with a Brüel and Kjaer measuring amplifier, using a Brüel and Kjaer 1/4 inch microphone placed in the position occupied by the bat's head. The spectral content of the tone bursts was examined by sending the output of the measuring amplifier through an analog-to-digital converter (National Instruments, model NB A2000) (sampling rate $500 \mathrm{kHz} ; 12$-bit resolution). The fast Fourier transform was then computed. Although significant harmonics were generated at frequencies below $7500 \mathrm{~Hz}$, because of the short rise/fall times, their magnitudes could not account for the ABR threshold except at the lowest frequency, $1250 \mathrm{~Hz}$. Although the $1250 \mathrm{~Hz}$ threshold is suspect, we nevertheless include it because it represents the minimum threshold value at this frequency. The actual ABR threshold may be higher at this frequency (see Fig. 3).

\section{Recording apparatus}

Sound-evoked electrical potentials were amplified (singleended) by a Dagan model 2400 extracellular amplifier (gain 5000-10000; bandpass 100-3000 Hz). The signals were further filtered by a Krohn-Hite bandpass filter $(100-2000 \mathrm{~Hz})$ and then sent to an analog-to-digital converter (National Instruments, model MIO-16-X) (gain 100; sampling rate $20 \mathrm{kHz}, 16$-bit resolution). The LabVIEW software computed, displayed and saved the averaged waveform from up to 512 stimulus presentations. An artifact suppression routine discarded any waveform containing spuriously high voltage levels, thus eliminating waveforms containing electrocardiograms and other muscle potentials.

\section{Experimental procedure}

ABR thresholds were measured at frequencies between 1.25 and $85 \mathrm{kHz}$. At each frequency, the sound level was reduced in $5 \mathrm{~dB}$ steps until a response was no longer observed. An averaged response was considered to exceed threshold if a peak or trough was present that matched one present at a higher intensity. 'Threshold' was then defined as the sound pressure level midway between the lowest level capable of eliciting a response and the highest level that failed to elicit a response. Within an experiment, several frequencies were tested repeatedly to assess the reliability of the response.

\section{Acoustic measurement of the outer ear}

\section{Data collection}

We measured the acoustic properties of the ear from three previously frozen specimens of $L$. borealis. Measurements were performed in a small room (approximately $2 \mathrm{~m} \times 1 \mathrm{~m} \times 1 \mathrm{~m}$ ) lined with sound-absorbing material to decrease acoustic reflections. A calibrated microphone (1/8 inch, Brüel and Kjaer, type 4138, protective grid removed) and a measuring amplifier (Brüel and Kjaer, type 2610) were used to measure sound pressure levels. After opening the bulla from below, the microphone was fitted to a plastic cone (Schlegel, 1977) whose tip was sealed with 
cyanoacrylate adhesive close to the position of the previously removed tympanic membrane (Coles et al. 1989; Jen and Chen, 1988). A sine-wave generator (Hewlett Packard, model 3594A sweeping local oscillator), a power amplifier (KrohnHite) and a custom-built electrostatic speaker $(2.5 \mathrm{~cm}$ in diameter) were used for sound production. The system had a flat frequency response $( \pm 3 \mathrm{~dB})$ between 15 and $125 \mathrm{kHz}$. Mounted on a freely movable arm, the speaker could be remotely positioned on a virtual sphere of $15 \mathrm{~cm}$ radius, centered at the position of the tympanic membrane (servo mechanism; Poussin and Schlegel, 1984; Schlegel et al. 1988). The frontal position of the bat (the assumed sonar direction) was aligned visually to $0^{\circ}$ elevation and $0^{\circ}$ azimuth. A photograph was taken of every preparation for later comparison with collected data (e.g. position of acoustic axis). The mechanical arrangement allowed for automated scanning of up to $260^{\circ}$ horizontally in steps of $2.00 \pm 0.25^{\circ}$ and of up to $160^{\circ}$ vertically in steps of $6.15 \pm 0.75^{\circ}$. Continuous pure tones from 5 to $125 \mathrm{kHz}$ were used in $5 \mathrm{kHz}$ steps with sound pressure levels of $80-120 \mathrm{~dB}$ SPL as test signals. To prevent measurements of spurious harmonics or noise, the signal registered by the microphone and amplified by the measuring amplifier was bandpass-filtered with a $3100 \mathrm{~Hz}$ bandwidth, centered at the testing frequency (Hewlett Packard, model $3590 \mathrm{~A}$ wave analyzer). The sound pressure was measured as voltage re $20 \mu \mathrm{Pa}$ with a 12-bit A/D converter board (Data Translation DT 2801A) installed in a microcomputer (Compaq Portable III). The same board also controlled the signal frequency (accurate to $\pm 1 \%$ ) and the servo mechanism, which executed the speaker movements. The automated measurement of 13 frequencies was accomplished in approximately $40 \mathrm{~min}$, which was a sufficiently brief period to avoid desiccation of the preparation. In contrast to freezing and thawing, desiccation would have major effects on the passive acoustic characteristics of the pinnae (Obrist et al. 1993). In one specimen, the pinna of the measured ear was then removed, and the same series of measurements was repeated. An adapted version of ASYST (ASYST Software Technologies) was used for data collection, analysis and display.

\section{Data analysis}

The sampled sound pressure values were stored in an array containing $26 \times 130$ data points. Further calculations were made using this array. The position of the peak indicated the acoustic axis of the ear at a given frequency. By subtracting the intensity value measured at this position in the pinna-deprived preparation from the value sampled in the intact ear, we determined the pinna-induced pressure gain. As a measure of the directionality of the sound pressure transformation, the angular width (azimuth) and height (elevation) of an isopressure contour line $3 \mathrm{~dB}$ below the peak was calculated and termed the '-3 dB acceptance angle' (Coles and Guppy, 1986).

We measured the characteristics of only one ear in each specimen, assuming that both ears are symmetrical relative to the mid-sagittal plane. By adding the original data array to a copy of itself, which was mirrored along the vertical central axis, we created a display of the total binaural directionality of hearing. Subtraction of the mirrored field from the original one creates a representation of the interaural intensity difference (IID), in some publications referred to as the interaural level difference (ILD). The azimuthal slope of these IID data (measured in degrees $\mathrm{dB}^{-1}$ at $0^{\circ}$ elevation and $0^{\circ}$ azimuth) indicates the binaural intensity change created when a sound source moves laterally. This value may be most important to the bat for lateralization of sound sources. Assuming a minimal binaurally detectable intensity difference of $1 \mathrm{~dB}$ (humans, cats, bats; Mills, 1960; Wakeford and Robinson, 1974; Witzke, 1989), this value may indicate detection thresholds for angular movement (Obrist et al. 1993).

Standing waves in the microphone-cone assembly created prominent fluctuations of the microphone sensitivity $( \pm 10 \mathrm{~dB})$ over the frequency range used. To allow comparisons between pressure differences as a function of frequency, we normalized each data array to the lowest value occurring therein. 'Peak pressures' mentioned in the text are therefore the highest values occurring in the 'normalized' data array and are not identical to the measured values, but rather code for the 'dynamic range' (highest minus lowest values) present in the data array.

\section{Echolocation signals}

Sonar signals of $L$. borealis were recorded in Pinery Provincial Park, Ontario, Canada, in the summer of 1986 (Obrist, 1995). Echolocation calls were recorded on a Racal Store 4D tape recorder operated at $76 \mathrm{~cm} \mathrm{~s}^{-1}$ using a QMC S200 microphone (sensitivity variation of $\pm 5 \mathrm{~dB}$ between 10 and $100 \mathrm{kHz}$ ). Slowed-down representative calls were analysed using CANARY (Cornell Laboratory of Ornithology, Ithaca, NY) on a Macintosh PowerPC 8500/120 computer for their spectral and temporal composition. Echolocation characteristics, hearing ability and pinna properties were thus recorded from bats of the same population.

\section{Results}

Different echolocation signals of Lasiurus borealis match spectrally to varying degrees with the sensitivity of ABRdeduced audiograms and with the acoustic performance of the pinnae. The recordings of convincing ABR waveforms in two red bats reveal a typically mammalian audiogram with good sensitivity between 10 and $50 \mathrm{kHz}$, peaking slightly below the spectral region of the sonar search calls. All passive properties of the ear show best tuning in the region between 40 and $50 \mathrm{kHz}$, where the ABR audiogram shows an unclear but conspicuous sensitivity peak and where echolocation calls are emitted during tracking and interception of prey. This raises some questions about the type of echolocation call for which the auditory system is optimized.

\section{Echolocation calls}

The design and general characteristics of the echolocation calls recorded were similar to previously published data 
Fig. 1. (A) Sonagram and power spectrum of search-, approachand final-buzz-phase echolocation calls of Lasiurus borealis. (B) Amplitude display of the same calls. The superimposed power spectrum in A displays the spectral energy of the underlying signal with frequency increasing towards the top (as in the underlying spectrogram) and intensity increasing towards the right.

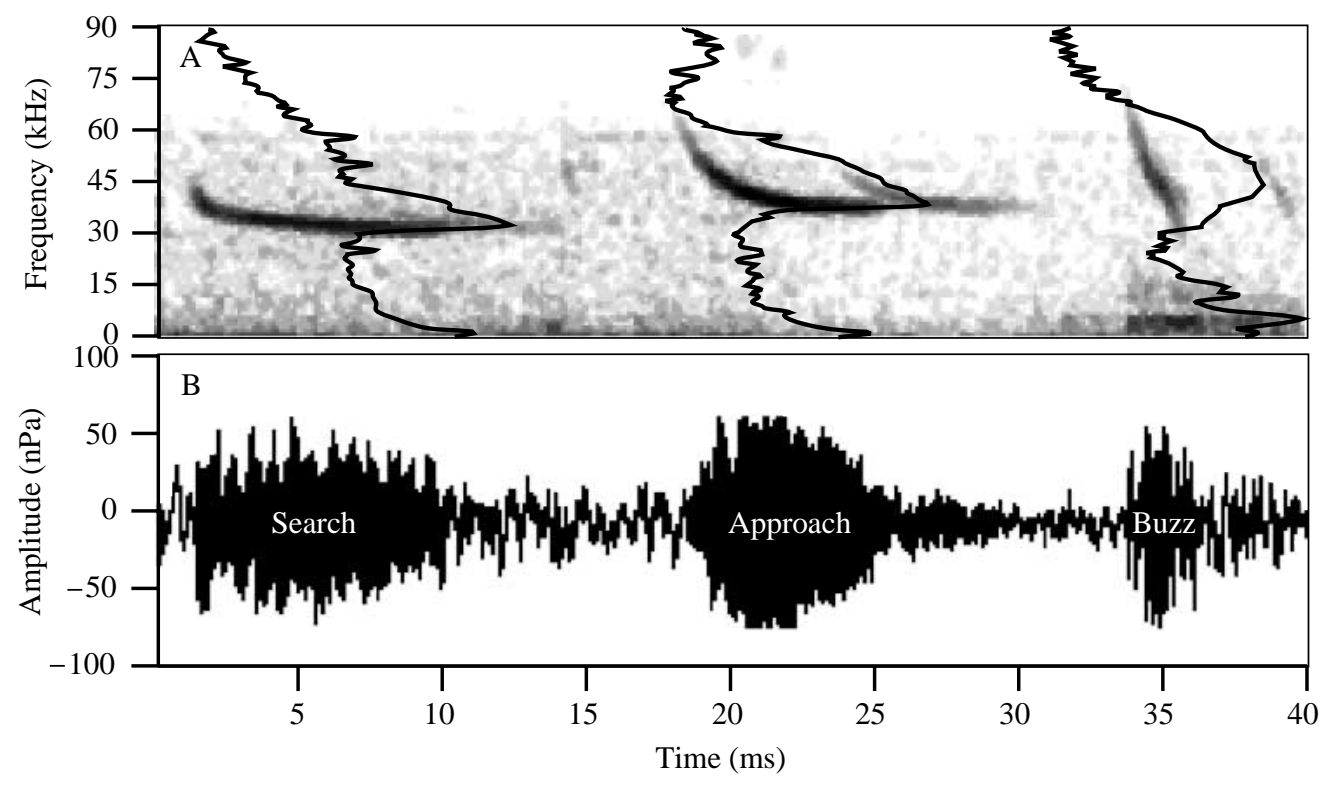

(Fenton and Bell, 1981; Griffin, 1958; Obrist, 1995). Orientation signals are dominated by sounds in the frequency band between 35 and $45 \mathrm{kHz}$. To relate these to our other results, we show the temporal and spectral composition of typical search-, approach- and final-buzz-phase echolocation calls (Fig. 1).

The fundamental sonar search calls sweep from 45 to $30 \mathrm{kHz}$, with maximum energy at $35 \mathrm{kHz}$. Approach-phase calls sweep from 65 to $35 \mathrm{kHz}$ with maximum energy at $40 \mathrm{kHz}$, and terminal-phase calls, sweeping from 70 to $30 \mathrm{kHz}$, show peak energy at $45 \mathrm{kHz}$ (Fig. 1).

\section{$A B R$ results}

ABR waveforms were comparable to those observed in other bats and in other mammals (Belknap and Suthers, 1982; Shaw, 1988; Wenstrup, 1984). Four positive peaks with latencies of approximately $1.3 \mathrm{~ms}(\mathrm{P} 1), 2.4 \mathrm{~ms}(\mathrm{P} 2), 3.9 \mathrm{~ms}(\mathrm{P} 3)$ and $6.4 \mathrm{~ms}$ (P5) were consistently observed in all waveforms obtained at high sound pressure levels (Fig. 2A). The latencies of these peaks clearly increased as the stimulus intensity was reduced and decreased as the stimulus frequency was increased. Even at low intensities, many of these major peaks could usually be observed (Fig. 2B). Near threshold, the existence of one or more peaks was verified in two independent ABR waveforms. These features support the view that the recordings were valid ABR waveforms.

ABR waveforms were frequency-dependent (Fig. 2A). At $30 \mathrm{kHz}$ and below, the four major peaks were evident, as well as a slow peak with a latency of approximately $15 \mathrm{~ms}$. At higher frequencies, there were as many a six peaks within the first $10 \mathrm{~ms}$, and the longer-latency slow peak occurred at latencies greater than $15 \mathrm{~ms}$. These peaks may reflect brainstem
Fig. 2. Auditory brainstem responses (ABRs). The shape of the ABR is dependent on frequency (A) and intensity (B). The $30 \mathrm{kHz}$ response at $32 \mathrm{~dB}$ SPL (B) was $5 \mathrm{~dB}$ above threshold. Amplitude units are arbitrary, but consistent in A and B. P1-P5, peaks 1-5; see text for futher details.
A

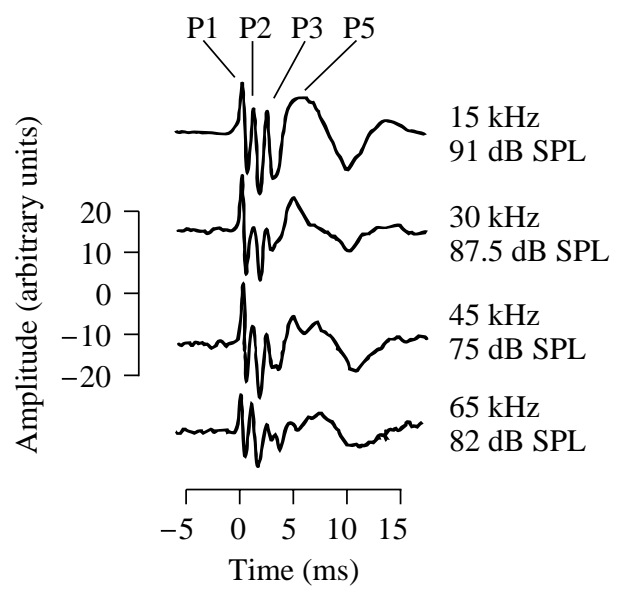

B
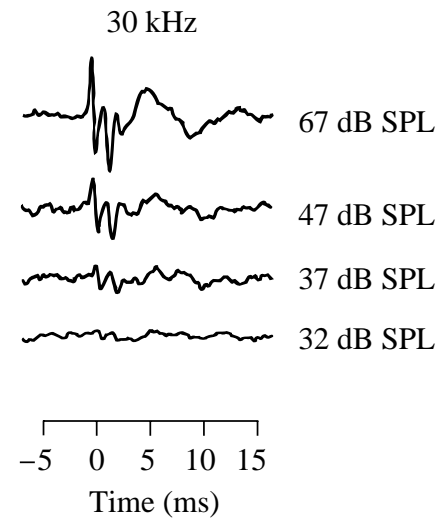
Fig. 3. ABR threshold curves of two Lasiurus borealis (A,B). The upper line in each figure shows the speaker response curve. Each symbol represents a threshold measurement. In some cases, more than one measurement was obtained for a particular test day. The lines connect measured values (if one measurement was obtained) or the mean of more than one measured value. Thresholds for bat 1 (A) were measured on two separate days. For bat 1 , the threshold measurement at $1250 \mathrm{~Hz}$ (labeled estimate) may be the result of harmonic distortion; the actual threshold may be higher (see Materials and methods).

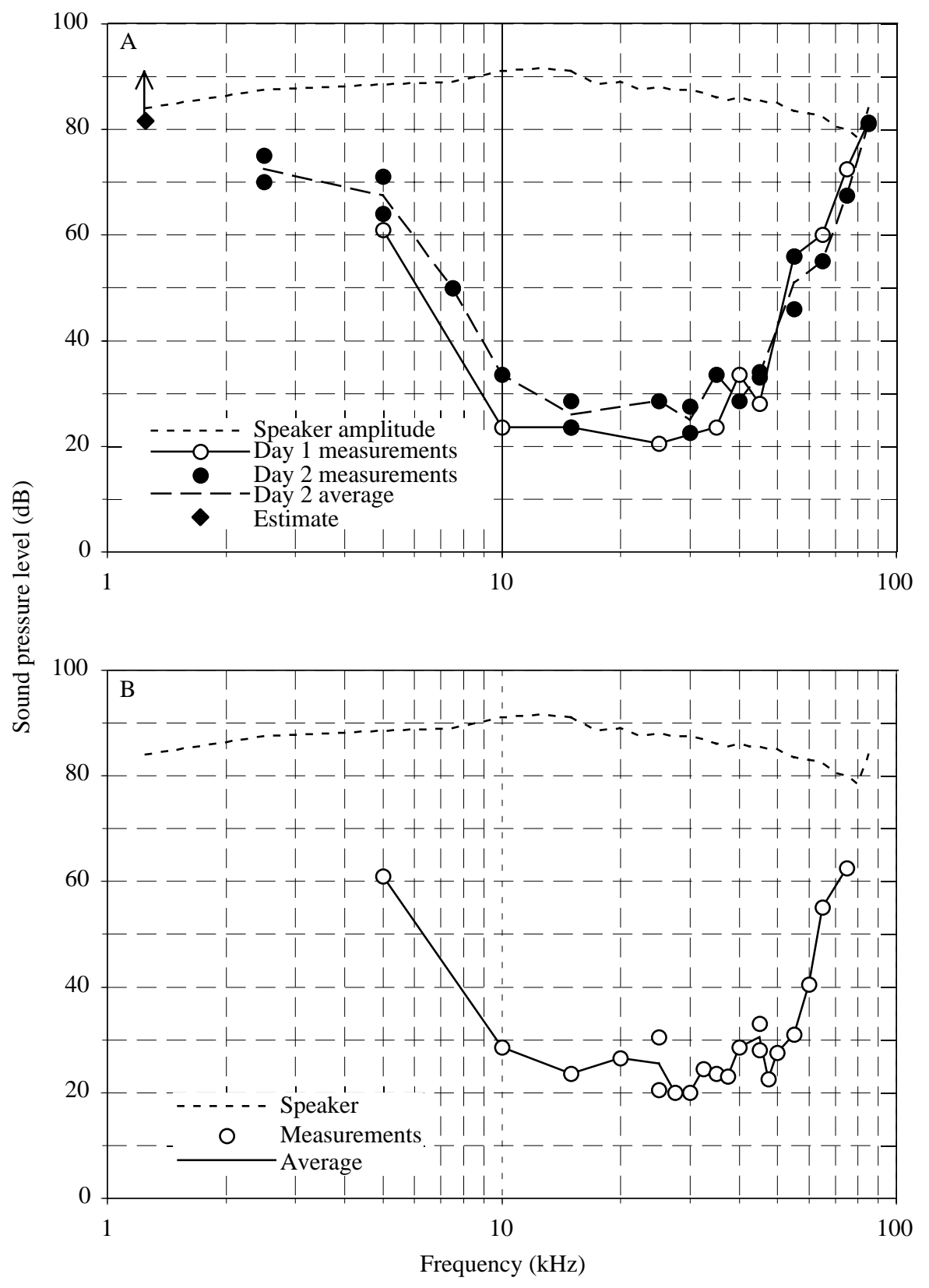

neuronal populations that are dominated by neurons tuned to particular frequency bands (Melcher and Kiang, 1996).

ABR threshold measurements (individual measurements, not mean values) for each animal at any frequency were relatively stable, never varying by more than $10 \mathrm{~dB}$ on the same or different days (Fig. 3A,B). In addition, ABR threshold measurements were similar for both animals. At only one frequency $(55 \mathrm{kHz})$ did ABR threshold measurements differ between the two bats by more than $10 \mathrm{~dB}$. Thus, the major features of the shape of the ABR audiogram were repeatable and similar across individuals. These features include a region of high sensitivity that extended from $10 \mathrm{kHz}$ to $45-55 \mathrm{kHz}$, with maximum sensitivity between 25 and $30 \mathrm{kHz}$. Here, thresholds were as low as $20 \mathrm{~dB}$ SPL. Below $10 \mathrm{kHz}$, sensitivity declined at a rate of approximately $35 \mathrm{~dB}$ per octave.
Above $45-55 \mathrm{kHz}$, sensitivity also declined sharply, at $50-70 \mathrm{~dB}$ per octave. At $85 \mathrm{kHz}$, only the maximum speaker intensity obtained a response; no frequencies above $85 \mathrm{kHz}$ were tested.

In each experiment, we noted a relative threshold minimum between 40 and $50 \mathrm{kHz}$, where the threshold at one test frequency was at least $5 \mathrm{~dB}$ lower than that for surrounding frequencies. More experiments are needed to determine whether this is a significant feature of the red bat's auditory sensitivity.

\section{Directional properties of the ear}

For one bat, the spatial dynamics of the measured sound pressure, of the IID and of pinna-related pressure effects are illustrated for 11 frequencies in Fig. 4. 'Peak pressures' 

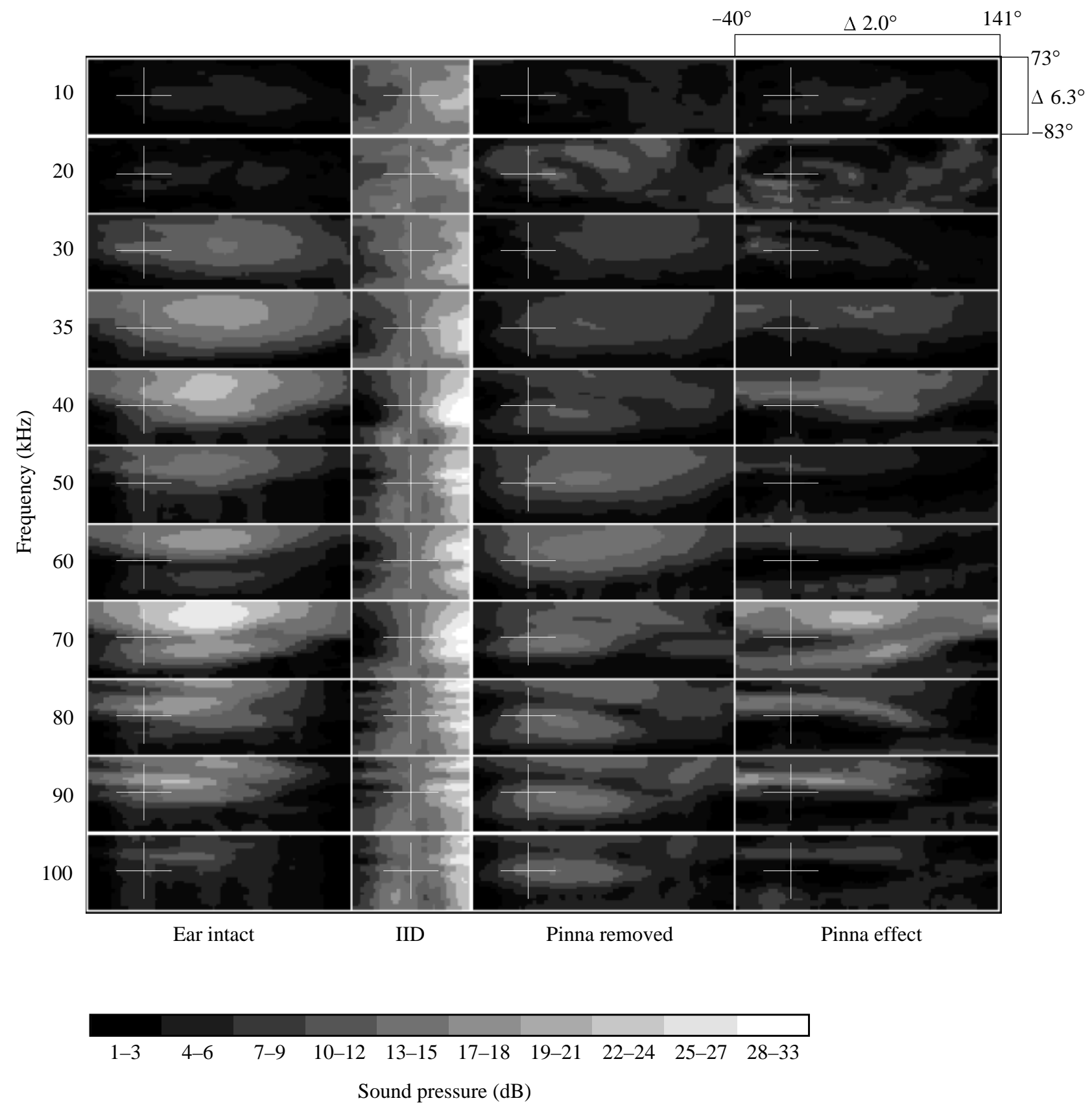

Fig. 4. Directionality of sound pressure transformation and derived data for Lasiurus borealis. For 11 frequencies (rows), the four columns show, from left to right: the sound pressure measured with the ear intact; the derived interaural intensity difference (IID); the sound pressure measured with the pinna removed; and the sound pressure increase effected by the pinna (the sound pressure of the intact ear minus the sound pressure of the pinna-removed specimen). Pressure values were normalized to the lowest value occurring per frequency and column. The frontal position of the preparation $\left(0^{\circ}\right.$ elevation; $0^{\circ}$ azimuth) was located at the position of the white cross; measurements were for the right ear. Sound pressure is coded in $3 \mathrm{~dB}$ steps, except for the $28-33 \mathrm{~dB}$ step $(6 \mathrm{~dB})$. Data were collected in increments of $2^{\circ}$ in the horizontal plane and $6.3^{\circ}$ in the vertical plane.

(defined for each frequency as the difference in sound pressure between the maximum and minimum sound pressures) increase with frequency, reaching a local maximum of $23 \mathrm{~dB}$ at $40 \mathrm{kHz}$ and a peak of $26 \mathrm{~dB}$ at $70 \mathrm{kHz}$ for the intact ear.

\section{Sharpness of directionality}

As a first-hand measure of the directionality of the sound pressure transformation of the pinna, we measured the height and width of the isopressure contour line $3 \mathrm{~dB}$ below the position of the peak pressure. This $-3 \mathrm{~dB}$ acceptance angle becomes smaller as ear directionality increases. Owing to decreasing wavelength, this value is expected to decrease with increasing frequency. However, both values show a sharp local minimum at approximately $40 \mathrm{kHz} \quad\left(41.7 \pm 7.3^{\circ}\right.$ width, 
Fig. 5. Mean width and height of $-3 \mathrm{~dB}$ acceptance angle ( \pm S.D., $N=3$ ), with an indication of the theoretical performance expected from a circular aperture of $12 \mathrm{~mm}$ (dashed line).
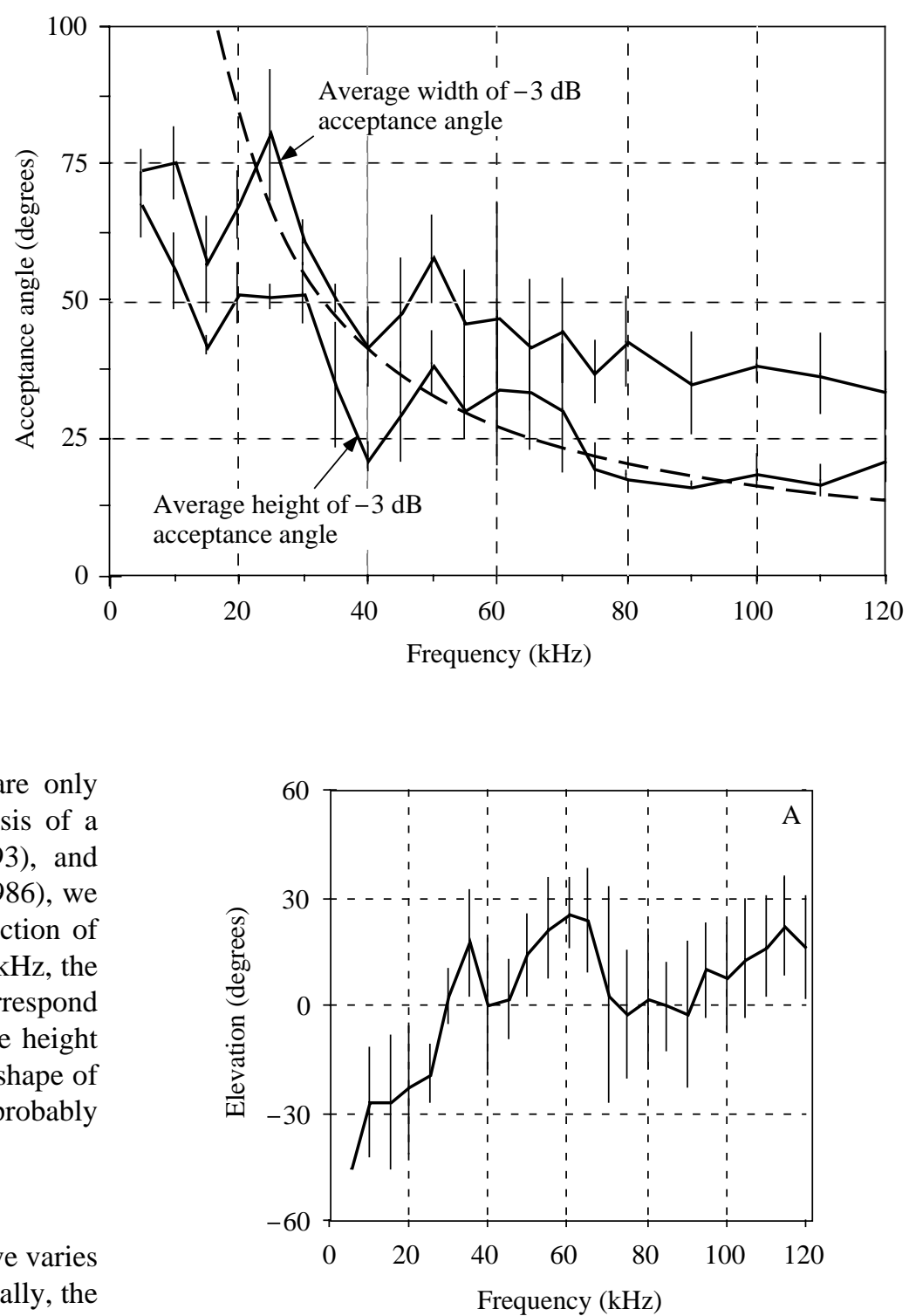

$21.3 \pm 2.6^{\circ}$ height; mean \pm S.D., $N=3$ ), values that are only approached again above $70 \mathrm{kHz}$ (Fig. 5). On the basis of a mean pinna diameter of $12 \mathrm{~mm}$ (Obrist et al. 1993), and following the example given by Coles and Guppy (1986), we calculated the theoretical acceptance angles as a function of frequency (dashed line in Fig. 5). Between 25 and $40 \mathrm{kHz}$, the width and height of the $-3 \mathrm{~dB}$ acceptance angle correspond well with these theoretical predictions, while only the height corresponds above this range. The slightly elongated shape of the pinna $(12.5 \mathrm{~mm}$ high, $11.5 \mathrm{~mm}$ wide $)$ is probably responsible for this effect.

\section{Acoustic axis}

The spatial location to which the ear is most sensitive varies with sound frequency, as in other bat species. Specifically, the location which produces the peak sound pressure at the ear the acoustic axis - increases in elevation as the frequency of sound increases (Fig. 6A). The azimuthal position of the acoustic axis moves towards the medial plane with increasing frequency (Fig. 6B). The position of the axis approaches the frontal position at $40-45 \mathrm{kHz}$, especially in elevation.

In the calculated, symmetrical, binaural directionality pattern (sound pressure for the measured ear added to that for the 'virtual' opposite ear: mirror image; see Materials and methods for details), we extracted the peak pressure at the central sagittal plane. This value indicates how loud a given sound source would appear binaurally and it increases with increasing frequency where the pinnae become more directional. The mean value for the three bats increases from $7 \mathrm{~dB}$ at $5 \mathrm{kHz}$ to an overall maximum of $16 \mathrm{~dB}$ at $40 \mathrm{kHz}$. After a reduction of $4 \mathrm{~dB}$, the pressures gradually increase, but never again reach the $40 \mathrm{kHz}$ peak value (Fig. 7).

\section{Pinna effect}

The influence of the pinna on ear directionality was

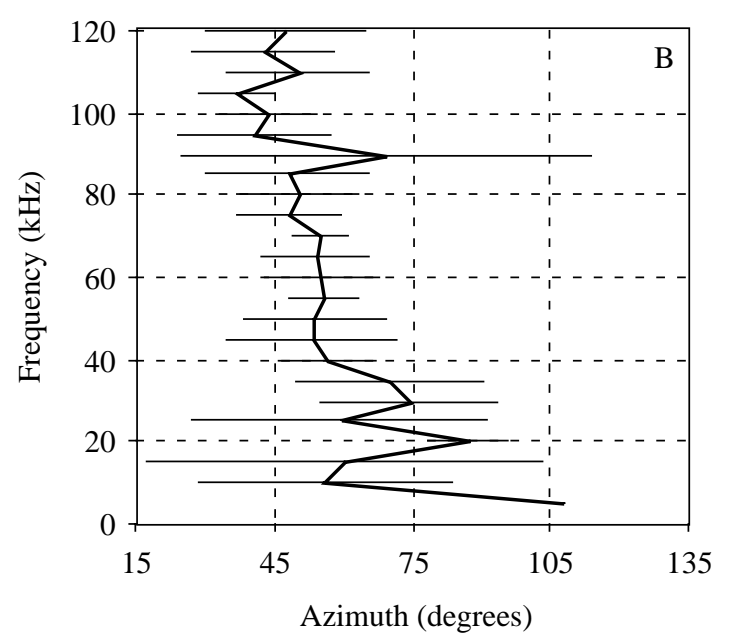

Fig. 6. Position of the acoustic axis (position of peak intensity) as a function of frequency. The mean ( \pm S.D.) elevation (A) and azimuth (B) of the axis are shown for three bats. 


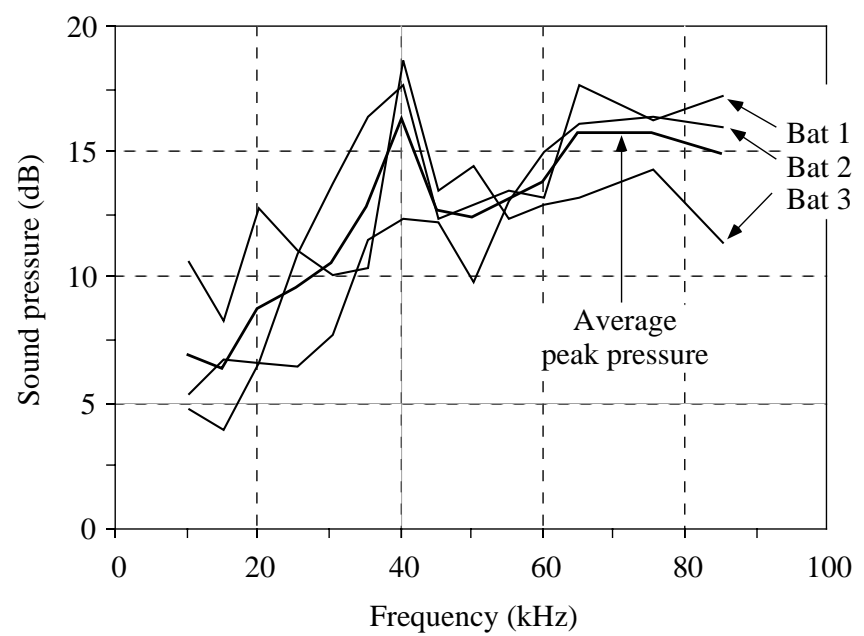

Fig. 7. 'Peak pressures' calculated for the binaural directionality pattern measured in three bats.

investigated in one specimen, in which we resampled the sound pressure transformation after removal of the pinna (Fig. 4, two columns on the right).

After having removed the pinna, the pressure peaks disappear and only a slight frequency-dependent increase in peak pressure (and directionality) remains. To calculate the actual pinna effect, the post-removal directionality patterns were subtracted from the pinna-intact patterns (Fig. 4, righthand column) and pressure values at the position of the acoustic axis were extracted (Fig. 8). The pinna contributes strongly to the directional sensitivity near $40 \mathrm{kHz}$ and again at $70 \mathrm{kHz}$. The pinna of $L$. borealis contributes to a peak sound pressure gain of $10 \mathrm{~dB}$ at $40 \mathrm{kHz}$.

\section{Interaural intensity difference}

By subtracting a copy of the original data field, mirrored along the vertical central axis, from the original data field, we

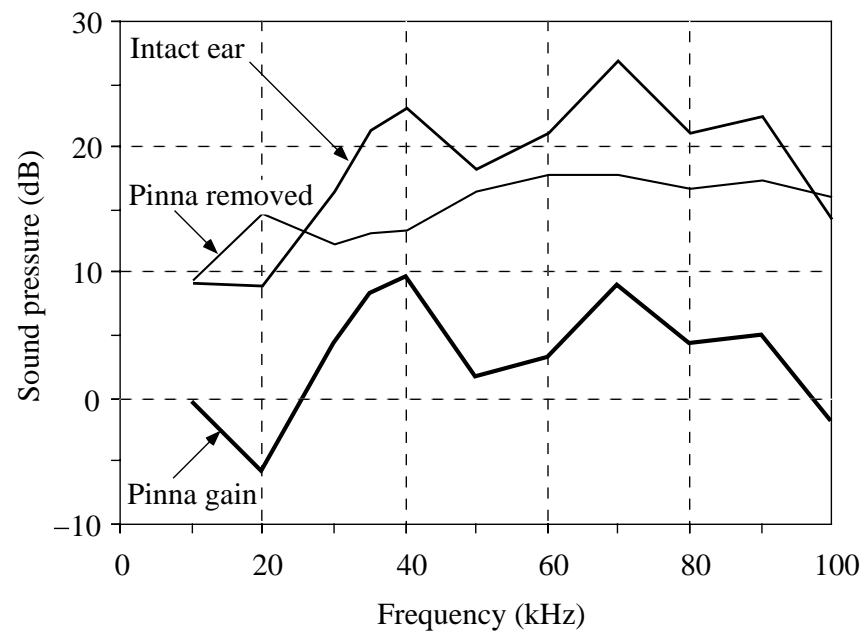

Fig. 8. Monaural sound pressure at the position of the acoustic axis with an intact ear and after removal of the pinna, and the net gain of the pinna.
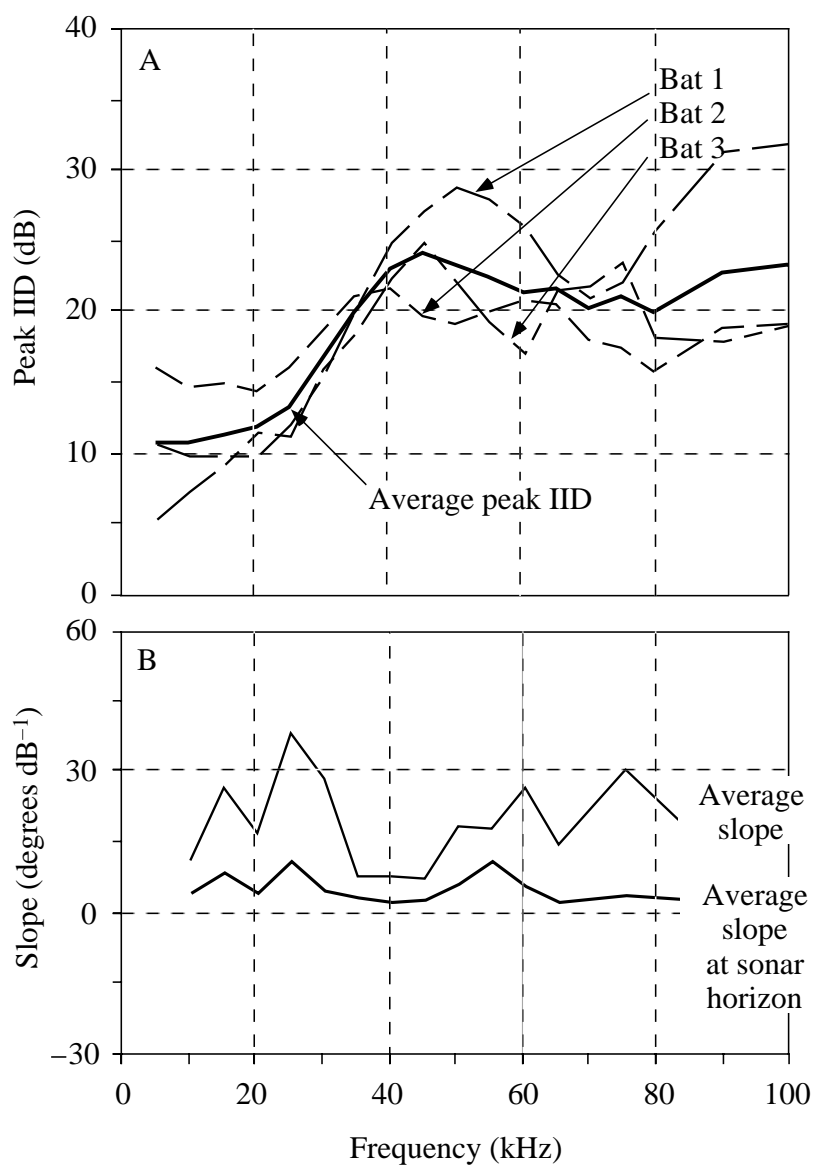

Fig. 9. Interaural intensity difference (IID). (A) The curves indicate the peak IID found at any given frequency for three individual bats and the mean values. Higher values indicate better cues for lateralization. (B) Slope of the azimuthal position versus IID function. When a right ear is measured, the IID rises from the left to the right hemisphere (compare Fig. 4, IID). The steepness of this slope at the sagittal plane (in degrees per $\mathrm{dB}$ ) indicates how far a sound source has to move laterally to elicit a binaural intensity difference of $1 \mathrm{~dB}$. Lower values indicate better cues for localization. The mean slope over all elevations and at the elevation of the sonar horizon are shown.

created a spatial representation of the interaural intensity difference (Fig. 4, IID column). We measured two features of frequency-dependant binaural intensity cues created by the two ears - the maximal interaural intensity difference (IID) and the slope of the azimuthal position versus IID function (Fig. 9). The maximal IID indicates the strength of intensity cues for lateralization. At low frequencies, it is relatively small because the small ears of L. borealis $(12.5 \mathrm{~mm} \times 11.5 \mathrm{~mm}$, Obrist et al. 1993) fail to achieve measurable directionality. At higher frequencies, the maximal IID rises to a mean value of $24 \mathrm{~dB}$ at $45 \mathrm{kHz}$ and is at least $20 \mathrm{~dB}$ at all higher frequencies (Fig. 9A).

The slope of the azimuth versus IID function indicates the strength of the interaural intensity difference cues in localization. Using the frontal sagittal plane as a reference, we calculated the number of degrees a sound source would have to move laterally to elicit an IID of $1 \mathrm{~dB}$ at the two ears (Fig. 9B). Averaged over three bats at all elevations, this varies 
from 8 to $30^{\circ} \mathrm{dB}^{-1}$, with a minimum of approximately $8^{\circ} \mathrm{dB}^{-1}$ at $35-45 \mathrm{kHz}$. We then calculated the change in the slope of the IID across the sonar horizon (the elevation of the acoustic axis at the frequency of the sonar signal; approximately $15^{\circ}$ in our preparation, see Fig. 6A). At this elevation, the values drop as low as $2.9^{\circ} \mathrm{dB}^{-1}$ at $40 \mathrm{kHz}$. Thus, directly in front of the hunting bat, a movement of a sound source of approximately $3^{\circ}$ will elicit an interaural sound pressure difference of $1 \mathrm{~dB}$ in L. borealis.

\section{Discussion}

This report describes aspects of hearing in the red bat and relates these to its well-described echolocation and foraging behavior (e.g. Acharya and Fenton, 1992; Balcombe and Fenton, 1988; Dunning et al. 1992; Hickey and Fenton, 1990; Obrist, 1995; Salcedo et al. 1995; Shump and Shump, 1982). Red bats use sonar signals in the range $30-70 \mathrm{kHz}$. ABR audiograms demonstrate peak sensitivity in the range $25-30 \mathrm{kHz}$. Measurements of the sound pressure transformation of the external ear show that the ear creates significant vertical and horizontal localization cues and that these cues are most pronounced at frequencies near $40 \mathrm{kHz}$. We believe that these properties may reflect adaptations related to the red bat's need to track sonar prey.

\section{Adaptations of vocalization and hearing in echolocating bats}

Echolocating microchiropteran bats that hunt airborne prey can be roughly separated by the principal characteristics of their calls and the way in which they interpret echoes. Rhinolophids, hipposiderids and one mormoopid species generally use long signals of high duty cycle with a strong constant-frequency (CF) component (for a review, see Neuweiler and Fenton, 1988). They separate pulse and echo spectrally. These long-CF bats show highly specialized auditory features related to their analysis of insect wingbeats, including very sharp cochlear tuning (Kössl and Vater, 1995) and expanded neural representations of their CF signals (Schuller and Pollak, 1979; Suga and Jen, 1976; Zook et al. 1985), reflecting the dominant frequency band of their echolocation signal. However, all other families rely on relatively short (generally $\leqslant 10 \mathrm{~ms}$ ) frequency-modulated signals with a low duty cycle (percentage sound duration of total time), which allow them to separate the outgoing pulse and returning echo temporally. While the signals of the latter group are considered Doppler-tolerant, the former group is capable of detecting the relative motion of either wings or whole prey items by detecting Doppler-shifted echoes.

A closer look at the latter group of echolocators reveals a variety of call types from very short and broad-band to rather long and narrow-band FM calls (e.g. Rhinopomatidae, Vespertilionidae, Molossidae; Neuweiler and Fenton, 1988). Some of these species make extensive use of very narrow-band signals, e.g. Rhinopoma hardwickei (Habersetzer, 1981), Tadarida macrotis and T. brasiliensis (Fenton and Bell, 1981; Simmons et al. 1978), Lasiurus cinereus (Belwood and
Fullard, 1984; Obrist, 1995) and L. borealis (Obrist, 1995). These signals guarantee successful long-range hunting owing to the concentration of most vocal energy in a narrow frequency band, which in most cases is additionally ideally suited for long-range signal transmission because of the relatively lower frequencies of these signals (Lawrence and Simmons, 1982). In addition, long signals would theoretically improve flutter detection, although Pipistrellus stenopterus is able to do this with steep FM signals (Sum and Menne, 1988).

Matching of auditory or pinna properties and echolocation behavior has been described for a variety of these FM species. Some Eptesicus fuscus can concentrate a large proportion of their sonar signal in a narrow frequency band when searching for targets, but less so than Lasiurus borealis (Obrist, 1995). In E. fuscus, the auditory threshold curve shows a minimum near $20 \mathrm{kHz}$ (Dalland, 1965) and, within the central auditory system, neurons in the inferior colliculus are especially sharply tuned to 20-30 kHz (Casseday and Covey, 1992) in the region of the narrow-band sonar signal $(22 \mathrm{kHz}$ low frequency and $27 \mathrm{kHz}$ main intensity; Obrist, 1995).

Habersetzer (1981) describes the dominant component of the very narrow-band sonar signals of Rhinopoma hardwickei as varying between 30 and $36 \mathrm{kHz}$, with single animals preferring 32-33 kHz. Simmons et al. (1984) report dominant frequencies around $36-40 \mathrm{kHz}$, and their recordings of evoked potentials of the midbrain show a clear sensitivity peak at $33-35 \mathrm{kHz}$. Finally, the ear characteristics of this species show a decrease in $-3 \mathrm{~dB}$ acceptance angles at $35 \mathrm{kHz}$ (Obrist et al. 1993).

The molossid Tadarida brasiliensis mexicana also uses narrow-band sweeps for echolocation. Considering the occurrence and abundance of this species, it is surprising to find differing reports on its predominant echolocation frequency, ranging from $50 \mathrm{kHz}$ (Simmons et al. 1978) to $27 \mathrm{kHz}$ (Fenton and Bell, 1981). This latter value corresponds well with the frequency band of maximal sensitivity in the audiogram $(21-27 \mathrm{kHz}$, Pollak et al. 1978) and with a region of expanded representation in the cochlea (Vater and Siefer, 1995). The authors call the expanded region an 'acoustic fovea' by analogy with the vastly greater specialization found in the CF bats, and they note that such specializations are generally found in the spectral range of high biological importance.

Three spectral ranges are meaningful for red bats; those utilized for social signals, those for search calls and those for approach and final calls, and our data underline their importance.

\section{ABR audiograms}

Frequency-dependent ABR thresholds usually provide a valid predictor of the shape of the audiogram (Shaw, 1988), but not necessarily of absolute auditory sensitivity (Belknap and Suthers, 1982). Behavioral thresholds in bats generally lie $20 \mathrm{~dB}$ or more below the measured ABR thresholds (Wenstrup, 1984). In studies of the fish-catching bat Noctilio leporinus, Wenstrup (1984) noted an exception to the close 
correspondence between the shapes of the ABR and behavioral audiograms, speculating that ABR thresholds may be lowered (relative to behavioral thresholds) at frequencies with disproportionately large auditory representations. However, that study used unusually low high-pass filtering of the ABR waveform $(1 \mathrm{~Hz}$ versus $10 \mathrm{~Hz}$ in the present study), which significantly changed the waveform, perhaps in a frequencydependent manner. If so, the shape of the ABR threshold curve could have been altered. The ABR filtering used in the present study corresponds more closely to that used in other species (Shaw, 1988). We thus suggest that the ABR threshold curves in the red bat more closely approximate the shape of the audiogram of this species than in the study of the fish-catching bat by Wenstrup (1984).

The ABR audiograms we measured in L. borealis match the spectra of its echolocation calls reasonably well, including feeding-buzz calls and long-range search-phase calls. The good sensitivity in the $10-30 \mathrm{kHz}$ range, below the sonar band, suggests that the red bat may use this band for social communication signals.

The local threshold minimum between 40 and $50 \mathrm{kHz}$, not coinciding with the dominant component of the search-phase echolocation calls, must be considered as tentative. We measured it in each experiment, but the effects we observed were relatively small and somewhat variable. In the big brown bat (Eptesicus fuscus), a species whose echolocation and foraging behavior resembles that of Lasiurus borealis, a similar local threshold minimum and maximum were found (Dalland, 1965; Koay et al. 1997). In that species, the local maximum has been related to the directional properties of the pinnae (Koay et al. 1997; Wotton et al. 1995). If the suggested local sensitivity peak in the red bat is supported by future studies, it would probably only play a role in the localization of approach and buzz pulses, which include these frequency bands.

\section{Sound pressure transformation of the external ear}

The sound pressure transformation of the ear indicates that it functions as an acoustic horn, becoming increasingly directional for wavelengths below $12 \mathrm{~mm}$, corresponding to a frequency of approximately $28 \mathrm{kHz}$ (Coles and Guppy, 1986; Coles et al. 1989; Fuzessery, 1996; Guppy and Coles, 1988). Owing to the asymmetric opening of the ear, the $-3 \mathrm{~dB}$ acceptance angles are different in elevation and azimuth. Furthermore, the position of the acoustic axis moves with decreasing wavelength towards the direction of the horn axis, indicated by the ear canal. The slight lowering and inward turn of the acoustic axis around $40-45 \mathrm{kHz}$ correlates with the tuning of the ear, as reflected by the decrease in the $-3 \mathrm{~dB}$ acceptance angles at $40 \mathrm{kHz}$. However, the significance and effect of this shift of the acoustic axis remain unclear.

The spectral range around $40 \mathrm{kHz}$ stands out in all our analyses of the ear's directional properties. Near $40 \mathrm{kHz},-3 \mathrm{~dB}$ acceptance angles show local minima while the directionality and the pinna gain reach their peaks. Thus, the ear is more sharply selective and provides the greatest gain near $40 \mathrm{kHz}$. The IID cues are also best near $40 \mathrm{kHz}$, where there is approximately $3^{\circ}$ change in azimuth for each decibel of IID. This slope of the IID change at the frontal position is an excellent predictor of lateralization performance in Megaderma lyra (Obrist et al. 1993; Witzke, 1989). Consequently, we predict that $L$. borealis exhibits best narrowband lateralization thresholds around $40 \mathrm{kHz}$.

\section{Hearing and hunting}

The pinnae of $L$. borealis show optimum sound-processing performance around $40-45 \mathrm{kHz}$. We suggest that an evolutionary pressure exists for improved hearing in the $40-45 \mathrm{kHz}$ region, where tracking calls are emitted, where the pinna performs best and where the audiogram exhibits a local minimum, rather than in the $30-35 \mathrm{kHz}$ region, where the species emits narrow-band search calls.

We think this pressure arises from the neccessity to improve tracking of prey during captures. Detection of a target is probably not critical for Lasiurus borealis. Red bats feed primarily on insects in the size range 5-20 mm (Hickey et al. 1996). In Nyctalus leisleri, a species that emits slightly lowerfrequency calls $(20-30 \mathrm{kHz})$ and exhibits a lower aspect ratio, simulated detection distances for targets of 5-20 mm varied between 5 and $15 \mathrm{~m}$ (Waters et al. 1995). This distance is reached in approximately $1-2 \mathrm{~s}$ by a red bat hunting at a mean flight speed of $6.7 \mathrm{~m} \mathrm{~s}^{-1}$ (Salcedo et al. 1995). Eptesicus fuscus detects a $4.8 \mathrm{~mm}$ sphere at $5.1 \mathrm{~m}$ (Kick, 1982), Pipistrellus sp. react to small moths 1.14-2.2 m before contact (Kalko, 1995; Kalko and Schnitzler, 1993). Therefore, 1-2 s is a reasonably long time for a hunting bat to decide on a target, when considering the $100-300 \mathrm{~ms}$ in which the actual catch is completed.

This final tracking and catching of the prey pose the greatest problem for the bat. Many of the moths preyed upon by $L$. borealis can hear the sounds that dominate their predators' echolocation calls (Fullard and Barclay, 1980; Fullard et al. 1983). The moths exhibit differing degrees of evasive maneuvers. Detection of faint search signals at long range elicits, if at all, a moderate 'flying-away' response in moths. However, upon hearing the louder and more frequent calls of an approaching bat, the moth will either emit clicks (Fullard, 1984) or avoid the bat by dropping or initiating a power-dive to the ground. At this moment, when the moth changes flight trajectory, the bat's localization performance becomes crucial. The fact that only $40 \%$ of all attacks are successful at the first attempt (Hickey and Fenton, 1990) illustrates the evasive success of the moths, which is only partly matched by the bat's ability to localize and maneuver to catch the evasive prey. Catching the prey on the first attempt is important. The eavesdropping capabilities of $L$. borealis (Balcombe and Fenton, 1988) illustrate the accuracy with which conspecifics locate failed attacks as well as their appreciation of the fact that, in $60 \%$ of all audible buzzes, the prey is still there!

Localization with short, broad-band buzz calls rather than detection with long narrow-band search calls stresses the 
spectral hearing region around $40-45 \mathrm{kHz}$, where the outer ear shows best performance, specifically the IID (which peaks at $45 \mathrm{kHz}$ ) and the optimum IID angular change at $35-45 \mathrm{kHz}$. By relating foraging behavior and ecology to the audiogram and the acoustic properties of the ear, we conclude that, in Lasiurus borealis, accurate localization of a sound source is more critical than detection of a target and has therefore put more evolutionary pressure on the shaping of the pinna and audiogram.

This research was made possible by a postdoctoral fellowship of the Swiss National Foundation for the Promotion of Science to M.K.O., an operating grant from NSERC to M. B. Fenton, the SFB 204 (Gehör) München, and a Biomedical Research Support Grant (National Institutes of Health) to J.J.W. The experiments reported here comply with the Principles of Laboratory Animal Care (NIH publication no. 85-23, revised 1985).

\section{References}

Acharya, L. AND Fenton, M. B. (1992). Echolocation behaviour of vespertilionid bats (Lasiurus cinereus and Lasiurus borealis) attacking airborne targets including arctiid moths. Can. J. Zool. 70, 1292-1298.

Balcombe, J. And Fenton, M. B. (1988). Eavesdropping by bats: the influence of echolocation call design and foraging strategies. Ethology 79, 158-166.

BelKnAP, D. B. AND Suthers, R. A. (1982). Brainstem auditory evoked responses to tone bursts in the echolocating bat, Rousettus. J. comp. Physiol. 146, 283-289.

Bell, G. P. And Fenton, M. B. (1984). The use of Doppler-shifted echoes as a flutter detection and clutter rejection system: the echolocation and feeding behaviour of Hipposideros ruber (Chiroptera: Hipposideridae). Behav. Ecol. Sociobiol. 15, 109-114.

Belwood, J. J. AND Fullard, J. H. (1984). Echolocation and foraging behaviour in the Hawaiian hoary bat, Lasiurus cinereus semotus. Can. J. Zool. 62, 2113-2120.

Casseday, J. H. ANd Covey, E. (1992). Frequency tuning properties of neurons in the inferior colliculus of an FM bat. J. comp. Neurol. 319, 34-50.

Coles, R. B. AND GupPY, A. (1986). Biophysical aspects of directional hearing in the tammar wallaby, Macropus eugenii. J. exp. Biol. 121, 371-394.

Coles, R. B., Guppy, A., Anderson, M. E. And Schlegel, P. (1989). Frequency sensitivity and directional hearing in the gleaning bat, Plecotus auritus (Linnaeus 1758). J. comp. Physiol. 165, 269-280.

Dalland, J. H. (1965). Hearing sensitivity in bats. Science 150, 1185-1186.

Dunning, D. C., Acharya, L., Merriman, C. B. And Dalferro, L. (1992). Interactions between bats and arctiid moths. Can. J. Zool. 70, 2218-2223.

Fenton, M. B. AND Bell, G. P. (1981). Recognition of species of insectivorous bats by their echolocation calls. J. Mammal. 62, 233-243.

Fenton, M. B., Swanepoel, C. M., Brigham, R. M., CebeK, J. And HICKEY, M. B. C. (1990). Foraging behaviour and prey selection by large slit-faced bats (Nycteris grandis; Chiroptera, Nycteridae). Biotropica 22, 2-8.
Fullard, J. H. (1984). Listening for bats: pulse repetition rate as a cue for defensive behaviour in Cycnia tenera (Lepidoptera; Arctiidae). J. comp. Physiol. A 154, 249-252.

Fullard, J. H. AND Barclay, R. M. R. (1980). Audition in spring species of arctiid moths as a possible response to differential levels of insectivorous bat predation. Can. J. Zool. 58, 1745-1750.

Fullard, J. H., Fenton, M. B. AND Furlonger, C. L. (1983). Sensory relationship between moths and bats sampled from two Nearctic sites. Can. J. Zool. 61, 1752-1757.

FuZESSERY, Z. M. (1996). Monaural and binaural spectral cues created by the external ears of the pallid bat. Hearing Res. 95, 1-17.

GRIFFIN, D. R. (1958). Listening in the Dark. Yale: Yale University Press.

Griffin, D. R., Webster, F. A. And Michael, C. R. (1960). The echolocation of flying insects by bats. Anim. Behav. 8, 141-154.

GUPPY, A. AND COLES, R. B. (1988). Acoustical and neural aspects of hearing in the Australian gleaning bats, Macroderma gigas and Nyctophilus gouldi. J. comp. Physiol. A 162, 653-668.

HABERSETZER, J. (1981). Adaptive echolocation sounds in the bat Rhinopoma hardwickei. J. comp. Physiol. 144, 559-566.

Henson, O. W., Bishop, A. And Keating, A. (1987). Biosonar imaging of insects by Pteronotus parnellii, the mustached bat. Nat. geog. Res. 3, 82-101.

Hickey, M. B. C., ACharya, L. AND Pennington, S. (1996). Resource partitioning by two species of vespertilionid bats (Lasiurus cinereus and Lasiurus borealis) feeding around street lights. J. Mammal. 77, 325-334.

Hickey, M. B. C. AND Fenton, M. B. (1990). Foraging by red bats (Lasiurus borealis): do intraspecific chases mean territoriality? Can. J. Zool. 68, 2477-2482.

JEN, P. H.-S. AND CHEN, D. (1988). Directionality of sound pressure transformation at the pinna of echolocating bats. Hearing Res. 34, 101-118.

KALKO, E. K. V. (1995). Insect pursuit, prey capture and echolocation in pipistrelle bats (Microchiroptera). Anim. Behav. 50, 861-880.

Kalko, E. K. V. And Schnitzler, H.-U. (1993). Plasticity in echolocation signals of European pipistrelle bats in search flight: implications for habitat use and prey detection. Behav. Ecol. Sociobiol. 33, 415-428.

KIcK, S. A. (1982). Target detection by the echolocating bat Eptesicus fuscus. J. comp. Physiol. 145, 431-435.

KoAy, G., Heffner, H. E. AND HeffNer, R. S. (1997). Audiogram of the big brown bat (Eptesicus fuscus). Hearing Res. 105, 201-210.

KÖSSL, M. AND VATER, M. (1995). Cochlear structure and function in bats. In Hearing by Bats, vol. 5 (ed. A. N. Popper and R. R. Fay), pp. 191-234. New York: Springer-Verlag.

LAWrence, B. D. AND Simmons, J. A. (1982). Measurements of atmospheric attenuation at ultrasonic frequencies and the significance for echolocating bats. J. acoust. Soc. Am. 71, 585-590.

Melcher, J. R. AND KIANG, N. Y. S. (1996). Generators of the brainstem auditory evoked potential in the cat. III. Identified cell populations. Hearing Res. 93, 52-71.

Mills, A. W. (1960). Lateralization of high-frequency tones. $J$. acoust. Soc. Am. 32, 132-134.

NeUweILER, G. AND Fenton, M. B. (1988). Behaviour and foraging ecology of echolocating bats. In Animal Sonar (ed. P. E. Nachtigall and P. W. B. Moore), pp. 535-549: New York, London: Plenum Press.

OBRIST, M. K. (1995). Flexible bat echolocation: the influence of individual, habitat and conspecifics on sonar signal design. Behav. Ecol. Sociobiol. 36, 207-219. 


\section{M. K. Obrist AND J. J. WenstruP}

Obrist, M. K., Fenton, M. B., Eger, J. L. And Schlegel, P. A. (1993). What ears do for bats: a comparative study of pinna sound pressure transformation in Chiroptera. J. exp. Biol. 180, 119-152.

Pollak, G. D., MArsh, D. S., Bodenhammer, R. And Souter, A. (1978). A single unit analysis of inferior colliculus in unanaesthetized bats: response patterns and spike count functions generated by constant frequency and frequency modulated sounds. J. Neurophysiol. 41, 677-691.

Poussin, C. And Schlegel, P. (1984). Directional sensitivity of auditory neurons in the superior colliculus of the bat, Eptesicus fuscus, using free field stimulation. J. comp. Physiol. A 154, 253-261.

RYDELL, J. (1993). Variation in the sonar of an aerial-hawking bat (Eptesicus nilssonii). Ethology 93, 275-284.

Salcedo, H. D., Fenton, M. B., Hickey, M. B. C. And Blake, R. W. (1995). Energetic consequences of flight speeds of foraging red and hoary bats (Lasiurus borealis and Lasiurus cinereus; Chiroptera: Vespertilionidae). J. exp. Biol. 198, 2245-2251.

SCHLEGEL, P. (1977). Calibrated earphones for the echolocating bat, Rhinolophus ferrumequinum. J. comp. Physiol. 118, 353-356.

SCHLEgel, P. A., Jen, P. H.-S. AND Singh, S. (1988). Auditory spatial sensitivity of inferior collicular neurons of echolocating bats. Brain Res. 456, 127-138.

SCHNITZLER, H.-U. (1987). Echoes of fluttering insects: information for echolocating bats. In Recent Advances in the Study of Bats (ed. M. B. Fenton, P. Racey and J. M. V. Rayner), pp. 226-243. Cambridge: Cambridge University Press.

SChnitzler, H.-U. AND Henson, O. W. (1980). Performance of airborne animal sonar systems. I. Microchiroptera. In Animal Sonar Systems (ed. R.-G. Busnel and J. F. Fish), pp. 109-181. New York, London: Plenum Press.

Schnitzler, H.-U., Menne, D., Kober, R. And Heblich, K. (1983). IV.2. The acoustical image of fluttering insects in echolocating bats. In Neuroethology and Behavioral Physiology (ed. F. Huber and H. Markl), pp. 235-249. Berlin, Heidelberg: Springer.

Schuller, G., Beuter, K. AND Hu, S. (1974). Response to frequency shifted artificial echoes in the bat Rhinolophus ferrumequinum. $J$. comp. Physiol. 89, 275-286.

Schuller, G. AND PollaK, G. (1979). Disproportionate frequency representation in the inferior colliculus of Doppler-compensating greater horseshoe bats: Evidence for an acoustic fovea. J. comp. Physiol. 132, 47-54.

Schumm, A., Krull, D. AND NeuweIler, G. (1991). Echolocation in the notch-eared bat, Myotis emarginatus. Behav. Ecol. Sociobiol. 28, 255-261.
SHAW, N. A. (1988). The auditory evoked potential in the rat - a review. Prog. Neurobiol. 31, 19-45.

Shump, K. A. AND Shump, A. U. (1982). Lasiurus borealis. Mammal. Spec. 183.

Simmons, J. A., Kick, S. A. And Lawrence, B. D. (1984). Echolocation and hearing in the mouse-tailed bat, Rhinopoma hardwickei: acoustic evolution of echolocation in bats. J. comp. Physiol. 154, 347-356.

Simmons, J. A., Lavender, W. A., Lavender, B. A., Childs, J. E., Hulebak, K., Rigden, M. R., Sherman, J., Woolman, B. And O'FARrell, M. J. O. (1978). Echolocation by free-tailed bats (Tadarida). J. comp. Physiol. 125, 291-299.

SugA, N. AND Jen, P. H.-S. (1976). Disproportionate tonotopic representation for processing CF-FM sonar signals in the mustache bat auditory cortex. Science 194, 542-544.

Sum, Y. M. AND MENNE, D. (1988). Discrimination of fluttering targets by the FM-bat Pipistrellus stenopterus? J. comp. Physiol. A 163, 249-354.

VATER, M. AND SIEFER, W. (1995). The cochlea of Tadarida brasiliensis: Specialized functional organization in a generalized bat. Hearing Res. 91, 178-195.

Von der EMde, G. AND MenNe, D. (1989). Discrimination of insect wingbeat-frequencies by the bat Rhinolophus ferrumequinum. $J$. comp. Physiol. A 164, 663-671.

WAKEFORD, O. S. AND RoBINSON, D. E. (1974). Lateralization of tonal stimuli by the cat. J. acoust. Soc. Am. 55, 649-652.

Waters, D. A., Rydell, J. AND Jones, G. (1995). Echolocation call design and limits on prey size: A case study using the aerial hawking bat Nyctalus leisleri. Behav. Ecol. Sociobiol. 37, 321-328.

Wenstrup, J. J. (1984). Auditory sensitivity in the fish-catching bat, Noctilio leporinus. J. comp. Physiol. A 155, 91-101.

WiTZKe, P. (1989). Acuity of pure tone lateralization by the Indian False Vampire, Megaderma lyra (Microchiroptera, Megadermatidae). In European Bat Research (ed. V. Hanák, I. Horácek and J. Gaisler), pp. 281-286. Praha: Charles University Press.

Wotton, J. M., Haresign, T., Ferragamo, M. J. and Simmons, J. A. (1995). Spatially dependent acoustic cues generated by the external ear of the big brown bat (Eptesicus fuscus). J. acoust. Soc. Am. 98, 1423-1445.

Zook, J. M., Winer, J. A., Pollak, G. D. And Bodenhamer, R. D. (1985). Topology of the central nucleus of the mustache bat's inferior colliculus: Correlation of single unit response properties and neuronal architecture. J. comp. Neurol. 231, 530-546. 